\title{
XIII.
}

Aus der I. Chirurg. Abtheilung des Krankenhauses Friedrichstadt zu Dresden (Med.-Rath Dr. H. Lindner).

\section{Ueber das erworbene Darmdivertikel und seine praktische Bedeutung.}

\author{
Von \\ Dr. C. Georgi, \\ Assistenzarzt der Abtheilung.
}

Während das angeborene sogenannte Meckel'sche Divertikel schon seit langer Zeit das Interesse der pathologischen Anatomen und Chirurgen in gleichem Maasse in Anspruch nahm, hat das erworbene Divertikel bis vor Kurzem eigentlich nur die Pathologen beschäftigt.

Erst durch die Mittheilungen Graser's auf dem Chirurgencongress 1898 ist die Aufmerksamkeit auch der Chirurgen auf das falsche Darmdivertikel und seine Folgezustände gelenkt worden. Es mehren sich auch seit dieser Zeit die Erfahrungen, durch die die praktische Bedeutung dieser Divertikel bewiesen wird.

Immerhin sind die einzelnen Mittheilungen noch spärlich, und so manches in dem ganzen Krankheitsbild noch unklar, so dass jeder hierher gehörige Fall der Veröffentlichung werth erseheint.

Ehe ich die Krankengeschichte eines Falles, der auf der ersten äusseren Abtheilung des Stadtkrankenhauses zu Dresden-Friedrichstadt vom Herrn Medicinalrath Dr. Lind ner beobachtet und operirt wurde, veröffentliche, sei es mir gestattet, einen ganz kurzen Ueberblick über die Entwicklung der Lehre von den falschen Darmdivertikeln zu geben, unter etwas genauerer Berïcksichtigung der seit den Mittheilungen Graser's veröffentlichten Fälle.

Das falsche Darmdivertikel war schon Morgagni bekannt; in der Mitte des 18. Jahrhunderts beschrieb dieser ein falsches Divertikel des Duodenums.

In Baillies "Anatomie vom krankhaften Bau" finden sich Beobachtungen über multiple Divertikelbildung am Darm. 
Rokitansky bezeichnet sie mit dem Namen "Schleimlıauthernien" mit Bezug auf ihren Durehtritt durch die Muscularis der Darmwand. Er führt bereits an, dass sie häufig eingedickte Fäcalmaterie enthalten und sie zuweilen der Sitz von Ulcerationen werden, die im retroperitonealen Bindenewebe Entzündungen hervorrufen können.

Klebs wies zuerst auf den Zusammenhang der Divertikelbildung mit der Gefässvertheilung am Darm hin.

Bei dem von ihm beobachteten Kranken, einem alten Manne, fanden sich am Dünndarm etwa 20 Divertikel, sämmtlich innerhalb des Mesenteriums, und zwar an solehen Stellen, an denen ein Gefässbündel vom Mesenterium auf den Darm übergeht, die kleinsten zwischen Arterie und Vene. Klebs versuchte dies dadureh zu erklären, dass bei fetten Individuen durch den Fettansatz im Mesenterium die Gefässe im Wachsthume zurückblieben und so einen Zug auf die Darmwand ausïbten. Er hielt sie also für Tractionsdivertikel.

Noch präciser als Klebs hat Hansemann den Zusammenhang der Divertikelbildung mit der Gefässvertheilung am Dünndarm durch ein genaues Studium der letzteren nachgewiesen. Nach ihm treten die Arterien vom Mesenterium auf die Darmwand über, verästeln sich unter der Serosa und senden ihre Aeste zur Muscularis und Submucosa; die entsprechenden Venen dagegen sammeln sich zwischen der Submucosa und inneren Muskelschicht und durchsetzen die Muscularis dicht am Mesenterialansatz, um hier in die Nähe der kleinen Arterien zu kommen. An dieser Stelle findet sich nun nach Hansemann um die Venenstämmchen eine Scheide von lockerem Bindegewebe, das mit einem an dieser Stelle ganz constanten Fettträubchen unter der Serosa zusammenhängt. Durch diese Venenscheiden treten nun auch die Divertikel hindureh.

Sehr interessant ist es, dass es Hansemann gelang - was schon Heschl vor ihm versucht hatte--, experimentell durch Wasseraufblähung verschiedener Därme namentlich bei senilen, schlaffen Personen an diesen typischen Stellen auch künstlich Divertikel zu erzeugen.

Den pathologischen Anatomen waren anch die seltenen Folgezustände der falschen Darmdivertikel schon lange bekannt, die durch die Kothstaunng in ihnen erzeugte Geschwürsbildung mit ansehliessender Perforationsperitonitis oder circumseripten Entzündung der Darmwand mit Stenosirung derselben.

Sidney Jones berichtete schon 1858 über einen 64 jährigen Mann, der Fäces mit seinem Urin entleerte und in Folge eines erschwerten Urindurehgangs durch einen Kothstein in der Blase starb. Die Section ergab multiple entzündete Divertikel der Flexura sigmoidea mit Communication zwischen Rectum und Blase.

Auch Fiedle ${ }^{1 /}$ ) beobachtete und beschrieb 1868 zwei einander sehr ähnliche Fälle von Divertikelbildung im Dickdarm mit Perforationsperitonitis und tödtlichem Ausgang, complieirt mit Phlegmone der Bauchdecken und Eitersenkung bis herab zur Glutaealmuseulatur. Bei der Section fanden sich namentlich in der Flexura sigmoidea zahlreiche zum Theil perforirte Divertikel.

Fiedler wies auch schon damals darauf hin, dass diese Divertikel namentlich im S. romanum gar nicht so sehr selten sind.

1) Denkschr. der Gesellschaf́t f. Natur- u. Heilkunde zu Dresden. 1864. 
„Wenn man bei Sectionen Erwachsener und namentlich älterer Leute die Wand des Colon und zumal des S. romanum genauer betrachtet, so findet man in derselben nicht selten mehr oder weniger ausgebildete Excavationen, die sich wesentlich und besonders durch ihre Kleinheit von den gewöhnlichen Dickdarmloculamenten unterscheiden."

'Trotzdem diese Folgezustände, die doch, wie man aus den Fie d l er'schen Fällen sieht, für den Praktiker von hoher Bedeutung werden können, schon so lange bekannt waren, ist das Interesse der Chirurgen auf diesen Gegenstand, wie oben erwähnt, eigentlich erst durch die Mittheilungen Graser's auf dem Chirurgencongress 1898 gelenkt worden.

Graser demonstrirte hier ein Präparat einer Stenose der Flexura sigmoidea, die durch Entzündung multipler falsclrer Divertikel bedingt war. Die Darmwand war dabei in einem Umfang von $5 \mathrm{~cm}$ Länge und $4 \mathrm{~cm}$ Dicke so derb infiltrirt, dass man Anfangs an ein Carcinom dachte; erst die intacte Schleimhaut und die unter- und oberhalb der Stenose bestehenden zahlreichen, zum Theil perforirten Divertikel erklärten das Krankheitsbild.

Durch diesen Fall angeregt, ging Graser den Ursachen der Divertikelbildung nach.

Vor ihm hatte schon Edel hervorgehoben, dass es sich hierbei wahrscheinlich um verschiedene Momente handele. Hauptsächlich sei es der durch die Kothstaung hervorgerufene erhöhte Druck, der bei Erschlaffung oder Atrophie der Darmwand an den dazu disponirten Stellen des Mesenterialansatzes (Gefässscheiden) die Divertikelbildung bedingte.

Grasel fand, dass die Divertikel am Dickdarm, was Hansemann schon für den Dünndarm nachgewiesen hatte, stets durch die Gefässlücken in der Musculatur hindurchtreten.

Er machte ferner die ganz merkwiirdige und überraschende Beobachtung, dass bei einem Drittel von Leichen, bei denen eine starke venöse Stauung: im Gebiete der Mesenterialvenen vorhanden war, im Bereich der Flexura sigmoidea mikroskopisch Divertikel nachzuweisen waren. Fr erklärte dies durch die in Folge der Stauung erweiterten Gefässlücken, wodurch eine grosse Prädisposition für Divertikelbildung gegeben sei. Zur Entstehung der Divertikel sei noch als Gelegenheitsursache namentlich chronische Obstipation mit Gasauftreibung hervorzuheben.

Entgegen dieser Annahme wies $\mathrm{Sudsuki}$ auf Veranlassung Hansemann's durch Untersuchung von 40 Leichen nach, dass ein bestimmter Zusammenhang zwischen Stauung in den Messenterialvenen und dem Auftreten von Darmdivertikeln nicht bestünde.

Seiner Meinung nach kommt es nicht auf die wechselnde Weite der Gefässe an, sondern vielmehr auf die Grösse und Nachgiebigkeit der bindeoder fettgewebigen Umgebung der Gefässe.

Ist diese reichlich entwickelt und schlaff, so ist die Disposition zur Divertikelbildung gegeben. Diese Disposition kann angeboren oder erworben sein.

Neben der von Graser als Gelegenheitsursache angeführten Kothstauung betonte $\mathrm{Sudsuki}$ auch die Muskelwirkung als dritten Factor in der Entstehung der falschen Darmdivertikel, indem diese die Schleimhaut durch die Gefässlücken an den Stellen des geringsten Widerstandes hiıdurchdrängen. 
Seit diesen Mittheilungen Graser's mehrten sich nun auch die in der Praxis beobachteten Fälle.

So veröffentlichte Rotter wenige Jahre nachher zwei dem Graserschen Falle ganz analoge Fälle von entzündlicher Strictur der Flexura sigmoidea.

Bei dem einen handelte es sich um einen 58jährigen Mann, der seit einiger Zeit Erscheinungen einer Darmstenose zeigte. Da in der Ileocoecalgegend ein kindskopfgrosser harter Tumor mit höckriger Oberfläche zu fühlen war, diagnosticirte man stenosirendes Carcinom des Coecums. Bei der vorgenommenen Operation bestätigte sich scheinbar die Diagnose. Man hielt den Tumor für ein Carcinom der nach rechts herübergeschlagenen Flexura sigmoidea und für inoperabel.

Erst die Section ergab als Ursache des Krankheitsbildes multiple Divertikelbildung, complicirt mit entzündlicher Stenose der Darmwand.

Der zweite von Rotter beschriebene Fall lag in der Zeit vor Graser's Veröffentlichungen.

Es handelte sich hier um einen 42 jährigen Mann, der in den letzten zwei Monaten an Stuhlbeschwerden litt und seit 3 Wochen Ileuserscheinungen zeigte.

Bei der Operation wurde ein Anus praeternaturalis angelegt, da eine Orientirung unmöglich war. 3 Tage nach der Operation trat der Exitus ein.

Bei der Section fand sich das S. romanum in einen klein faustgrossen harten Tumor verwandelt, der mit der Blase und der Beckenwand verwachsen war. Auch hier glaubte man selbst noch bei der Section Anfangs ein Carcinom vor sich zu haben; erst die genauere Untersuchung ergab, dass ein entzündlicher Process vorlag mit zahlreichen Fistelgängen in der Darmwand und Stenose derselben.

Rotter hält es für sehr wahrscheinlich aus dem ganzen pathologischanatomischen Befund, dass auch hier der Krankheit Divertikelbildung zu Grunde lag. Leider wurde, da man die Graser'schen Veröffentlichungen noch nicht kannte, nicht darauf geachtet.

Ferner sind ganz kürzlich zwei Fälle von Perforationsperitonitis in Folge Durchbruchs eines ulcerirten Divertikels der Flexura sigmoidea veröffentlicht worden.

In dem einen Falle von Mertens handelte es sich um einen 45jährigen Mann, der angeblich erst seit 8 'Tagen über Schmerzen in der Unterbauchgegend und Stuhlverstopfung klagte. In Folge starken Pressens bei der Defäcation traten stärkere Schmerzen auf und im Ansehluss daran die Zeichen der Perforationsperitonitis.

Bei der Operation fand sich allgemeine eitrige Peritonitis. Der Ausgangspunkt konnte nicht gefunden werden. Zwei Tage nachher Exitus.

Bei der Section fand sich als Ausgangspunkt der Peritonitis die Perforation eines falschen Darmdivertikels am unteren Ende der Flexura sigmoidea. Die Perforation erfolgte hier im Anschluss an das starke Pressen bei der Defäcation.

Einen zweiten Fall beschreibt Hilbert.

Ein 37 jähriger Mann litt schon seit 6 Monaten an den Erscheinungen einer Herzinsufficienz. Ganz allmählich ohne alarmirende Symptome stellten sich die Zeichen einer Perforationsperitonitis ein. Der Verlauf war nahezu 
schmerzlos und mit Diarrhoen complicirt, wahrscheinlich erklärbar aus der hochgradigen Stauung in Folge seines Herzleidens.

Auch hier wies die Section die Perforation eines falschen Darmdivertikels im unteren Theil der Flexura sigmoidea nach.

Einen Fall von Perforation eines ulcerirten Divertikels in die Blase, ähnlich dem oben schon erwähnten, im Jahre 1858 von Sidney Jones bereits beschriebenen, hat kürzlich Waldvogel aus der Ebstein'schen Klinik publicirt.

Ein Arzt erkrankte mit 35 Jahren unter merkwürdigen Symptomen, Schmerzen in beiden Beinen, Beschwerden beim Uriniren und beim Stuhlgang. Die Krankheit zog sich 16 Jahre hin.

Nach 15jährigen Bestehen derselben trat plötzlich Durchbruch in die Blase ein. Der Urin wurde trüb, es traten Gas aus der Harnröhrenmündung, beim Uriniren und später Fäces. Das Allgemeinbefinden litt unter dem Bestehen der Fistel verhältnissmässig wenig. Der Patient erlag einem Herzleiden.

Die Section ergab Verwachsungen der Flexura sigmoidea mit der hinteren Blasenwand. In diesen Verwachsungen fand sich ein Abscess, der durch 2 perforirte falsche Darmdivertikel mit dem Darmlumen und durch einen Fistelgang mit der Blase communicirte.

Das gleichzeitige Vorkommen von falschen Darmdivertikeln und Carcinom beobachtete Hochenegg; er exstirpirte ein Carcinom an einer Flexura sigmoidea, die zahlreiche bis kirschgrosse Divertikel zeigte.

Er stellte sich die Entwickelung dieses Carcinoms auf dem Boden eines ulcerativen Processes in einem dieser Divertikel vor.

Die zuletzt erwähnten Fälle betrafen sämmtlich die Flexura sigmoidea. Den an anderen Stellen des Darmtractus vorkommenden falschen Divertikeln scheint nicht dieselbe Bedeutung zuzukommen.

Moser berichtet über ein falsches Divertikel des Coecums, das man gelegentlich einer Herniotomie im Bruchsack fand.

Hier sei auch noch der sehr interessante Fall von Payr erwähnt, von dem es allerdings dahingestellt bleibt, ob es sich um ein angeborenes oder erworbenes Divertikel handelt.

Der dünne Stiel eines durch Entzïndung in einem Leistenbruch fixirten Divertikels war allmählich durch die fortwährenden Darmbewegungen so ausgezogen worden, dass schliesslich eine völlige Isolirung dieses Divertikels im Bruchsack eintrat. In Folge Retention entzïndlicher Producte brach dieses Divertikel durch die änssere Haut durch. Es fand sich in der äusseren 
obachteten und operirten Fall von Divertikelbildung an der Flexura sigmoidea zu veröffentlichen.

v. B., Generalmajor, 64 Jahre, aufgenommen am 21. Juni 1901.

Patient, erblich nicht belastet 1) und früher stets gesund, litt seit einigen Jahren an Stuhlbeschwerden, Verstopfung abwechselnd mit Durchfällen; seit 2 Monaten beträchtliche Verschlimmerung der Beschwerden: Stuhl selten und wenig, nur auf Einlauf; dabei kolikartige Schmerzen in der linken Unterbauchgegend.

Die Untersuchung des grossen, kräftigen, nicht abgemagerten, aber etwas blass aussehenden Mannes ergab Folgendes:

Herz: Töne rein, Grenzen normal; Puls 80, regelmässig. - Mässige Arteriosklerose des peripheren Gefässsystems.

Lungen: normal.

Abdomen wegen reichlichen Fettpolsters schwer zu palpiren. Jueber nicht vergrössert. In der linken Unterbauchgegend findet sich eine undeutliche druckempfindliche Resistenz. Die Untersuchung in Narkose ergab hier einen nicht scharf umschriebenen, unbeweglichen Tumor von derber Consistenz und höckriger Oberfläche. Die Bauchdecken darüber waren normal.

Das Rectum war frei; im Stuhl kein Eiter, kein Blut.

Urin $\mathrm{OB}$.

Die Diagnose wurde mit einer gewissen Wahrscheinlichkeit auf Carcinom der Flexura sigmoidea gestellt.

24. Juni. Operation in Morphium-Aethernarkose und Beckenhochlagerung. Schnitt durch linken Rectus unterhalb des Nabels. Keine Zeichen einer Peritonitis; Colon descendens etwas gebläht.

Die Flexura sigmoidea ist in einen faustgrossen, derben, mit der Beckenund vorderen Bauchwand verwachsenen Tumor verwandelt.

Wegen unlösbarer Verwachsungen wurde von einer Exstirpation des vermeintlichen Carcinoms abgesehen.

Der Wundverlauf war normal; der Eingriff verlief ohne wesentliche Folgen. Am 27. Juni bekam Patient wegen Kolikschmerzen Glycerinsuppositorium; danach Stuhl. Um sich zu reinigen, ein Wärter war momentan nicht zur Stelle, steht Patient auf, wurde ohnmäehtig und stürzte sehr schwer aufschlagend zu Boden. Nach der sofortigen Aufhebung tiefster Collaps. Sofort reichliche Excitantien. 28. Juni. Collaps überwunden; aber heftige Schmerzen im Leib, Erbrechen, Stuhl und Windverhaltung; dabei Gesicht verfallen, Abdomen etwas anfgetrieben, überall druckempfindlich; Puls 120, klein, Temperatur 37,60. 29. Juni. Exitus unter den Symptomen allgemeiner Peritonitis.

Bei der Section zeigte sich die Operationswunde reactionslos.

Die Flexura sigmoidea fand sich mit der Becken- und vorderen Bauchwand ziemlich fest verwachsen. In ihrem unteren Drittel fand sich eine für einen Finger eben durchgängige ca. $3 \mathrm{~cm}$ lange Strictur. Der Dickdarm oberhalb derselben war etwas gebläht.

1) Er gab allerdings an, dass sein Vater, ein in hohem Alter verstorbener Arzt, an sich selbst eine Darmstenose lange vor seinem Tode diagnosticirt, und zwar genau localisirt habe. Die auf Wunseh des Verstorbenen ausgefïhrte Section ergab die Richtigkeit der Diagnose. 
Die Schleimhaut innerhalb der Stenose und oberhalb derselben ist ziemlich stark geschwollen, schiefriggrau verfärbt; auch sieht man hier mehrere erbsengrosse, mit kurzem Stiel aufsitzende, schwärzlich verfärbte Polypen; ausserdem einzelne pfennigstückgrosse, flächenhafte Schleimhautdefecte mit unregelmässig zackigen Rändern.

Oberbalb und unterhalb der Stenose finden sich mehrere Ausbuchtungen der Darmwand, in die man die Kuppe des kleinen Fingers hineinlegen kann, zum Theil mit intacter Schleimhaut ausgekleidet, theilweise auch mit geschwürigem Grunde.

Von einem dieser Divertikel an der medialen Seite der Flexur gelangt man in eine in der Darmwand zwischen Muscularis und der stark verdickten Serosa gelegenen Abscesshöhle, die ca. $5 \mathrm{~cm}$ lang und $2 \mathrm{~cm}$ breit, mit dickem, graugrünen Eiter erfüllt ist.

Diese Höhle steht mit mehreren, aber kleineren ähnlich in der Darmwand gelegenen Abscessen durch zahlreiche Fistelgänge in Verbindung, so dass die stark verdickte Serosa im ganzen Bereich der Flexura sigmoidea durch diese Abscesse und Fistelgänge unterminirt erscheint.

Eine dieser Höhlen, welche aber schon extraperitoneal gelegen ist, communicirt durch eine linsengrosse Perforationsstelle mit der Bauchhöhle.

Die Serosa der übrigen Därme zeigt lebhafte Gefässinjection und theilweise zarte Fibrinauflagerungen. Eiter oder Koth sind in der Bauchhöhle nicht zu finden.

Leber, Nieren, Milz zeigen keine Stauung.

Der vorliegende Fall ist dadurch interessant, als er die Folgeerscheinungen der erworbenen Darmdivertikel an der Flexura sigmoidea in ausserordentlich klarer Weise zur Anschauung bringt.

Wir sahen hier neben einer hochgradigen Stenose des Darmrobres eine sehr umfangreiche, beinahe im ganzen Bereich der Flexur sich findende, dissecirende Eiterung innerhalb der Darmwand, verbunden mit Perforation eines der Abscesse und nachfolgender Peritonitis.

Dass diese Eiterung in diesem Umfang möglich war, ist lediglich erklärbar durch die zahlreichen Verwachsungen und die starke Verdickung der Serosa.

Der durch die gleichzeitig bestehende Stenose stark vermehrte innere Druck im Darmrohr hat dem Eiter den Abfluss in's Darmlumen ersehwert und ihn förmlich in das subseröse Gewebe hineingepresst.

Eine plötzliche starke Anspannung der Bauchpresse hat dann ähnlich dem Mertens'schen Fall die Perforation herbeigeführt.

Klinisch standen in dem Krankheitsbilde unseres Falles die Stenoseerscheinungen in dem Vordergrund. Hervorzuheben wäre, dass trotz der ausgedehnten Eiterung verhältnissmässig geringe Schmerzen und nie Fieber bestand.

Bei der Gesammtbetrachtung der seit der Mittheilung Graser's 
veröffentlichten, hierher gehörigen Fälle fällt es zunächst auf, dass sie fast sämmtlich die Flexura sigmoidea betrafen.

Den hier vorkommenden Divertikeln scheint auch im Vergleich zu den an anderen Darmabschnitten beobachteten eine gewisse Sonderstellung zuzukommen.

Graser fand auch in Uebereinstimmung hiermit die Anfangsstadien dieser Divertikel, die nur mikroskopisch nachweisbaren herniösen Ausstiulpungen der Schleimhaut durch die Muscularis, fast ausschliesslich in der Flexura sigmoidea.

Eine Erklärung für diese Erscheinung ist bisher noch nicht gefunden, man muss sich vorläufig begnügen mit der Annahme, dass die Kothstauung und die Gasauftreibung - wohl die wesentlichsten Factoren in der Entstehung der erworbenen Darmdivertikel - in der Flexura sigmoidea am stärksten wirken.

Bei der relativen Häufigkeit dieser mikroskopisch nachweisbaren Divertikel - Graser fand unter 28 mikroskopisch untersuchten Darmstücken von Leichen mit Stauung in den Unterleibsvenen diese multiple Divertikelbildung in zehn Fällen - ist es auffallend und bisher noch völlig unerklärt, warum die obigen Folgezustände so ausserordentlich selten sind.

Vielleicht sind diese Divertikel öfter, als man bisher annahm, der Ausgangspunkt der an der Flexura sigmoidea relativ häufig beobachteten partiellen Peritonitis mit Adhäsionsbildung. Doch fehlen diesbezügliche Untersuchungen noch ganz.

Sind einmal entzündliche Erscheinungen in diesen Divertikeln aufgetreten, so scheinen sie verhältnissmässig häufig zur Stenose zu führen, in den obigen 7 Fällen 5 mal.

Seltener tritt Perforation dieser Divertikel in die freie Bauchhöhle ein; es treten meist vorher beträchtliche Verwachsungen in der Umgebung ein; es kommt zur circumscripten Peritonitis mit Abscessbildung, die ibrerseits wieder in die freie Bauchhöhle oder in Hohlorgane, Blase u. s. w. perforiren können. Was die klinischen Erscheinungen anlangt, die von den erworbenen Divertikeln der Flexura sigmoidea ausgehen, so sind sie verschieden je nach dem pathologisch anatomischen Substrat.

In der Mehrzabl der Fälle, falls keine stärkeren entzündlichen Erscheinungen in ihnen vorhanden sind, machen sie gar keine Symptome.

Erst wenn diese hinzukommen, treten zeitweise geringe Schmerzen in der Gegend der Flexur auf, die namentlich bei Obstipation stärker werden. 
Diese Gegend ist dann meist auch druckempfindlich; in den ausgeprägten Fällen kann man dann einen harten Tumor fühlen.

Hat die Entzündung zur Stenose oder Perforation geführt, so beherrschen die davon ausgehenden Symptome das Krankheitsbild.

Noch auf einen Umstand, der von grosser praktischer Bedeutung ist, möchte ich hier hinweisen.

Der durch die chronische Entzündung bedingte Tumor ist, wie die obigen Fälle zeigen, fast immer mit Carcinom verwechselt worden, nicht allein, wie es uns erging, während der Operation, sondern in mehreren oben angeführten Fällen selbst noch während der Section, so dass erst die mikroskopische Untersuchung zur richtigen Diagnose führte.

Man gehe wohl nicht mit der Annahme fehl, dass ein grosser Theil dieser Fälle früher gar nicht diagnosticirt oder fälschlicher Weise für Carcinom erklärt wurden.

Durch obigen Fall angeregt, habe ich die Protokolle der in den Jahren 1891-1901 in der pathologisch-anatomischen Abtheilung des Stadtkrankenhauses Dresden-Friedrichstadt secirten Leichen auf Divertikelbildung hin durehgesehen.

Während bei 8133 Leichen $8 \mathrm{mal} \mathrm{Meckel'sche} \mathrm{Divertikel} \mathrm{sich}$ fanden, waren erworbene Divertikel $7 \mathrm{mal}$ notirt. Der Sitz der Letzteren war nur in einem Falle der Dünndarm, in allen übrigen 6 Fällen der Dickdarm. Leider ist nicht angegeben, wie viel davon auf die Flexura sigmoidea fallen.

Stärkere Entzündungserscheinungen sind, abgesehen von unserem Fall, in keinem der übrigen beobachtet; alle anderen bildeten nur einen Nebenbefund bei der Section.

Stärkere Stauung in den Unterleibsorganen in Folge Herz- oder Lungenleiden war nur in 2 Fällen notirt; einen Zusammenhang zwischen Staung und dem Auftreten erworbener Divertikel ergab also diese Zusammenstellung nicht; ebensowenig liess sich Kachexie als disponirende Ursache nachweisen; 3 von den 7 Leichen waren im Gegentheil sehr kräftig und gut genährt.

Aus obigen Zahlen einen Schluss auf die Häufigkeit dieser Divertikel zu ziehen, will ich mir nicht erlauben, da, wie wir ja gesehen haben, diesem Krankheitsbild erst in neuerer Zeit das ihm gebührende Interesse entgegen gebracht wurde.

Zum Schluss gestatte ich mir, meinem hochverehrten Chef, Herrn Medicinalrath Dr. Lindner, für die Anregung zu dieser Arbeit meinen Dank auszusprechen. 